\title{
A qualitative model of the Daniell cell for chemical education
}

\author{
Paulo Salles \\ University of Brasília, Institute of Biological Sciences, Campus Universitário Darcy Ribeiro \\ Asa Norte, 70.910-900, Brasília, Brasil \\ psalles@unb.br \\ Ricardo Gauche \\ University of Brasília, Institute of Chemistry, Campus Universitário Darcy Ribeiro \\ Asa Norte, 70.910-900, Brasília, Brasil \\ gauche@unb.br \\ Patrícia Virmond \\ University of Brasília, Institute of Chemistry, Campus Universitário Darcy Ribeiro \\ Asa Norte, 70.910-900, Brasília, Brasil \\ patriciavirmond@bol.com.br
}

\begin{abstract}
Understanding how students learn chemical concepts has been a great concern for researchers of chemical education. This community is making efforts to identify the most important misunderstandings and develop strategies to overcome conceptual problems. Qualitative Reasoning has great potential for building conceptual models that can be useful for chemical education. This paper describes a qualitative model of the Daniell cell, to support understanding of interactions between chemical reactions and electric current. The model support explanations about changes in the mass of the electrodes, a bulb attached to the system that goes on and off and about changes in the colour of the cathode solution. Finally, we discuss the potential of qualitative articulate models for science education of deaf people.
\end{abstract}

\section{Introduction}

Why does the colour of copper sulphate changes when the Daniell cell is functioning? Any Brazilian student in a secondary school should be able to answer this question, given that the Daniell cell is largely used to build up concepts on the relation between chemical reactions and electric current. Teachers try to bring this knowledge into everyday life, as it explains the functioning of a number of home devices. Electrochemistry became an important area for the development of chemical reasoning among secondary school students. However, the students can hardly give a causal account of the mechanisms working on the Daniell cell typical behaviour. Communication between the student and the teacher about electrochemistry is difficult. Textbooks are widely used in Brazilian schools (for example, Hartwig et al. 1999), but they communicate a simplistic view of chemistry and do not contribute for the development of fundamental concepts, including those in electrochemistry (Lopes, 1992). The laboratory is not an option in this case, because even when building apparently simple batteries, experiments in general do not work very well. Computer models and simulations are interesting alternatives for supporting the development of a conceptual framework of electrochemistry. However, are they actually being used by the teachers? Ribeiro \& Greca (2003) reviewed papers published in 15 leading international journals of chemical education during a period between 1992 and 2002 and showed that the use of software is increasing, but it is still far from expectations. The authors found 74 references to models and simulations in their survey. In 67 , the simulations were used to create mental representations and to build conceptual understanding of chemical knowledge. Only 16 were effectively being used in classroom (13 at the university level and only 3 in secondary schools). It is worth to note that the survey did not find any use of authoring tools or of modelling tools, showing that teachers that are using chemical educational software hardly develop their own didactic material (Ribeiro \& Greca, 2003). Qualitative Reasoning (QR) has great potential for developing conceptual models that could be used in science education (for an example in ecology, see Salles et al. 2003). The potential of QR models in chemical education was explored by Mustapha et al. (2002), who describe a system for simulating a chemistry laboratory. Using the ontology provided by the Qualitative Process Theory (Forbus, 1984), the lab supports secondary school students in qualitative analysis of chemical compounds and in simulations of chemical reactions. Here we describe the implementation of a qualitative model for students to understand the structure and behaviour of the Daniell cell. This model is meant to be explored by deaf students as part of the project "Português como segunda língua na educação científica de surdos" (Portuguese as second language in the scientific education of the deaf) (cf. Salles et al., 2004). Section 2 describes details of the problem to be modelled and raise some of the most relevant requirements of electrochemistry education. Section 3 describes the design and implementation of a qualitative model. Section 4 describes a simulation and the results obtained. The paper ends in section 5, with remarks about ongoing work and the potential of QR modelling for chemical education. 


\section{The Daniell cell}

The Daniell cell consists of a zinc rod dipping in a solution of zinc sulphate, connected by a wire to a copper rod dipping in a copper sulphate (II) solution. Spontaneous oxidation and reduction reactions generate electric current, with electrons passing from the zinc rod to the wire and from it to the copper rod, originating a current along the wire. During the functioning of the battery the following transformations are observed: the zinc rod goes under corrosion and its mass reduces; consequently, concentration of ions $\mathrm{Zn}^{2+}$ increases in the half cell; the copper rod receives a deposit of molecules of metal and its mass increase. Consequently, the concentration of ions $\mathrm{Cu}^{2+}$ in the solution decreases. The functioning of the battery can be demonstrated by a bulb (that shows electric current passing through the wire) and by the colour of the solution in the cathode cell. Dissolved in water copper sulphate produces a blue coloured solution. During the functioning of the battery, this substance disappears and the liquid becomes colourless.

\section{The modelling process}

The process centred approach (Forbus, 1984) was chosen because it allows for the explicit representation of objects, quantities, possible values, processes and qualitatively distinct states in which the system can be found. The models were implemented in GARP (Bredeweg, 1992). Relevant properties of the objects are represented as quantities. Qualitative values of quantities are given by the tupple $<$ magnitude, derivative $>$. The magnitude represents the amount of a quantity (for example, the concentration of a substance is small) and the derivative represents its direction of change (that is, the quantity is increasing, stable or decreasing). Quantity spaces (QS) used in this model include \{colourful, colourless\} for the quantity colour_of_solution; \{zero, positive $\}$ for the quantity electric current; $\{$ on,off $\}$ for the quantity light. The QS \{plus\} was used for the quantities mass of metal rods, ions in the solutions and potential in the electrode. For all the quantities, derivatives draw on the QS $\{+, 0,-\}$. Causality is represented by direct influences and qualitative proportionalities. The former represents processes, the primary cause of change. If, for example, $\mathrm{I}+(\mathrm{A}, \mathrm{B})$, then the value of $\mathrm{B}$ is added to the magnitude of $\mathrm{A}$ and, as a result, $\mathrm{A}$ starts to increase or decrease (if $B$ is negative). Proportionalities propagate to other quantities changes caused by processes. In this case, the causal link is established via the derivatives. If $\mathrm{P}+(\mathrm{A}, \mathrm{B})$ and the derivative of $B$ changes, it causes the derivative of $A$ to change in the same direction. For example, if $\mathrm{B}$ is increasing $([\delta \mathrm{B}=+])$ then $\mathrm{A}$ is also increasing $([\delta \mathrm{A}=+])$. The condition for the functioning of the battery is the existence of a difference of potential between the two electrodes, being in the initial state the potential at the anode greater than the potential at the cathode. This difference results in the process 'generation of electric current'. This process poses direct influences on the mass of the metals that will be further propagated to the rest of the system, like the bulb and the solutions in both cells (see Figure 1).

\section{Simulations and results}

The simulation with this model presented here generates only two states: the initial state (state 1), that lasts some time interval without relevant qualitative changes and the final state (state 2), referring to a situation when the battery is no longer functioning. In state 1 , the potential in the anode is greater than the potential in the cathode. This situation creates a flow of electrons (electric current) that leave the rod with greater potential and move to the rod with lower potential, setting the value on to the bulb connected to the wire. The ordered rate of flow of electrons results in an increasing mass at the electrode with smaller potential, and decreasing mass of the electrode with a higher potential. Variations in the mass of the metals also affect the potential of the electrodes. Where the mass grows (cathode), the amount of ions in the solution decreases (as they move to the metal rod) and the potential of the electrode (the metal rod in the cathode) increases. On the other half-cell, loss of mass of the metal rod results in increased concentration of ions in the solution and decreased potential of the electrode (the anode). This situation leads to a state transition. In state 2 , the process stops because there is no longer difference of potentials between the electrodes (the chemical equilibrium), and the battery does not work. The bulb is off and the copper sulphate solution becomes colourless (Figure $1)$.

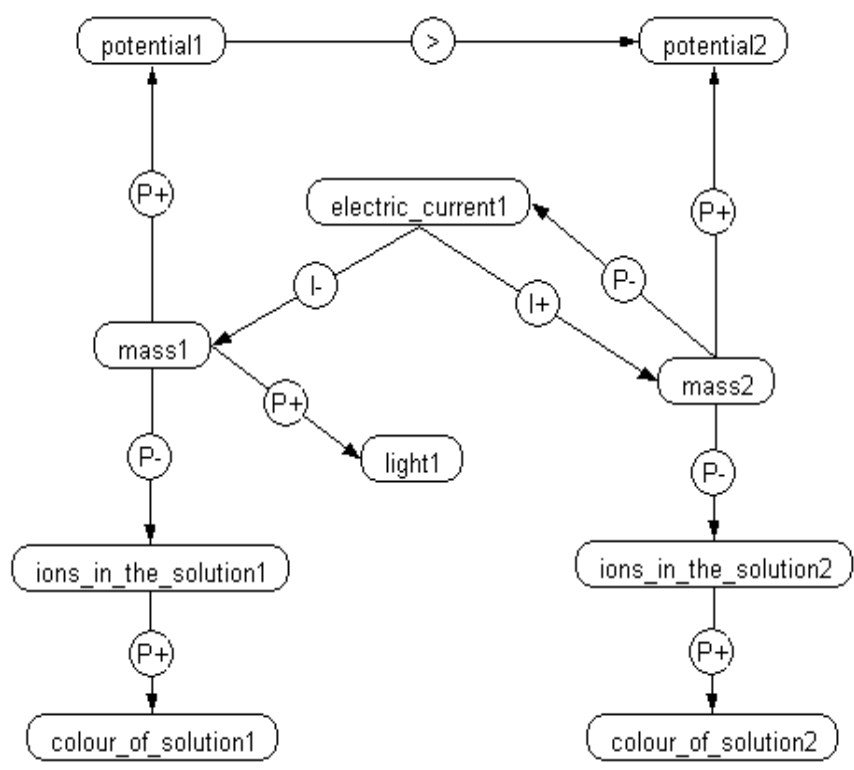

Figure 1: Dependencies between quantities in state 1 of a simulation..

\section{Discussion and final remarks}

This work describes a qualitative model of the Daniell cell. Normally textbooks limit explanation to the transformation 
of the chemical energy into electric energy, but they do not describe how these transformations happen. A QR approach has an added value because it focus on the causal relations that determine the behaviour of the cell. A description of the mechanism of change, the electric current generation process, indicates the origin of the dynamic phenomenon, which is then propagated to and observed in the rest of the system. This way, inspecting only the causal model of the battery the student can explain why the mass of rods change, and why the bulb goes off while the colour of the solution at the cathode disappears. However, QR has potential to impact other areas of science education not covered so far. The work described here is part of an umbrella project (see below) that aims at the development of Portuguese as a second language for deaf students, whose native language is LIBRAS (Brazilian Sign Language). The use of qualitative models to support second language acquisition by deaf students is already being investigated and the results obtained so far are encouraging. Salles et al. (2004) found in an experiment that students exposed to qualitative models were consistent in the ability of recognizing objects and processes, building up causal chains and applying them to a given situation, assessing derivative values of quantities and making predictions about the consequences of changes in a system. The students were successful in writing up a composition about an ecological accident, using linguistic descriptions of the relevant physical and social processes. Ongoing work includes exploring the qualitative model of the Daniell cell with a group of deaf students in an experiment similar to the one described in Salles et al. (2004), improved by the lessons learned.

\section{Acknowledgments}

The work described here was partially funded by the project "Português como segunda língua na educação científica de surdos" (Portuguese as second language in the scientific education of the deaf), a grant MEC / CAPES / PROESP from the Brazilian government.

\section{References}

Bredeweg, B. (1992) Expertise in Qualitative Prediction of Behaviour. Ph.D. thesis, University of Amsterdam, Amsterdam, The Netherlands, 1992.

Forbus, K.D. (1984) Qualitative process theory. Artificial Intelligence, 24.

Lopes, A. R. C. (1992) Livros didáticos: obstáculo ao aprendizado da ciência Química. Química Nova, 15(3).

Mustapha, S.M.F.D.; Jen-Sen, P. \& Zaim, S.M. (2002) Application of Qualitative Process Theory to qualitative simulation and analysis of inorganic chemical reaction. In: N. Agell \& J. A. Ortega (Eds.) Proceedings of the International workshop on Qualitative Reasoning, $\left(Q R^{\prime} 02\right)$, pages 177-184, Sitges - Barcelona, Spain, June 10-12, 2002.

Ribeiro, A.A. \& Greca, I.M. (2003) Simulações computacionais e ferramentas de modelização em educação química: uma revisão de literatura publicada. Química Nova, 26(4). 